\title{
Methyl 8-Acetyl-labdanolate \\ (-)-(3S)-5-((1R,2R,4aS,8aS)-2-Acetoxy-2,5,5,8a-tetramethyldecahydro-1-naphthalenyl)-3- methylpentanoic Acid Methyl Ester
}

\section{Juan M. Castro, Sofia Salido, Joaquin Altarejos*, Manuel Nogueras and Adolfo Sanchez}

Departamento de Química Inorgánica y Orgánica, Facultad de Ciencias Experimentales, Universidad de Jaén, 23071 Jaén, Spain

Tel.: 34-953-002743, fax: 34-953-012141, E-mail: jaltare@ujaen.es

Received: 12 December 2001 / Accepted: 19 September 2002 / Published: 8 March 2003

Keywords: Diterpenes, labdanes, methyl labdanolate, acetylation, tertiary alcohol

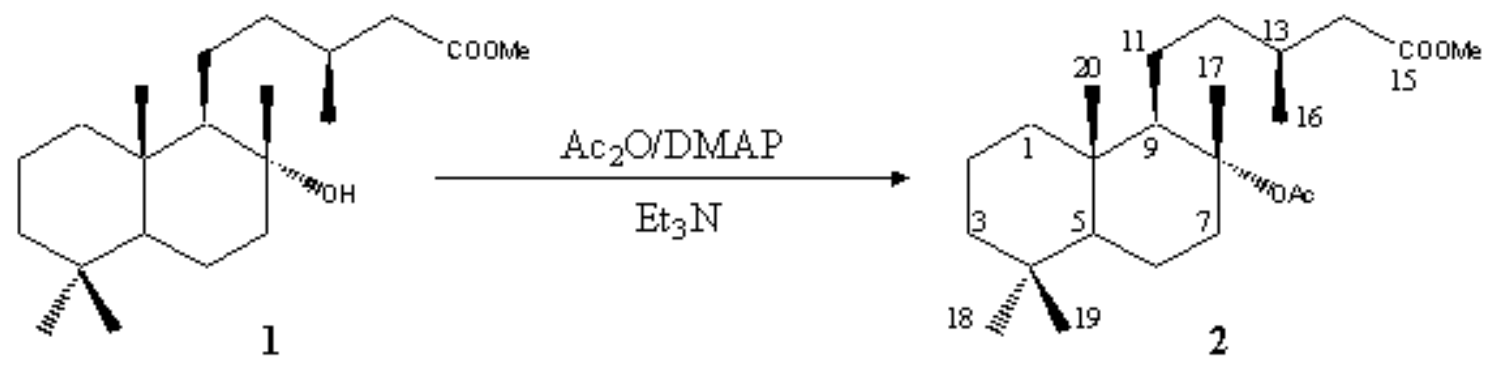

Acetic anhydride $(0.53 \mathrm{~mL}, 5.61 \mathrm{mmol})$ and $N, N$-dimethylaminopyridine $(17 \mathrm{mg}, 0.14 \mathrm{mmol})$ were added to a stirred solution of the alcohol $1(442 \mathrm{mg}, 1.31 \mathrm{mmol})$ in freshly distilled triethylamine $(1.3 \mathrm{~mL})$ [1]. The reaction was mantained between $35-40{ }^{\circ} \mathrm{C}$ for $72 \mathrm{~h}$ and, then, water was added $(25 \mathrm{~mL})$ and the mixture extracted with $\mathrm{Et}_{2} \mathrm{O}(3 \times 40 \mathrm{~mL})$. The combined organic layers were washed with $2 \mathrm{~N} \mathrm{HCl}(25$ $\mathrm{mL})$, saturated $\mathrm{Na}_{2} \mathrm{CO}_{3}(25 \mathrm{~mL})$ and brine $(3 \times 25 \mathrm{~mL})$. The organic phase was dried over anhydrous $\mathrm{Na}_{2} \mathrm{SO}_{4}$ and the solvent evaporated under reduced pressure to yield a residue $(539 \mathrm{mg}$ ) which was purified by flash chromatography on silica gel, using a 3:2 hexane/Et $2 \mathrm{O}$ mixture as eluent, to give the pure title compound 2 (296 mg, $0.78 \mathrm{mmol}, 60 \%$ ).

Mp: 81.0-82.3 ${ }^{\circ} \mathrm{C}$ (white crystals, from hexane).

$[\mathrm{a}]_{\mathrm{D}}=-31.8^{\mathrm{o}}\left(\mathrm{c} 0.97 \mathrm{cg} \cdot \mathrm{mL}^{-1}, \mathrm{CHCl}_{3}\right)$.

IR (neat, $\mathrm{n}, \mathrm{cm}^{-1}$ ): 1735 (COOMe), 1723 (OAc), 1252, 1217, 1148 (COOMe, OAc).

${ }^{1} \mathrm{H}$ NMR (300 MHz, $\left.\mathrm{CDCl}_{3}, \mathrm{~d}, \mathrm{ppm}\right): 0.78\left(3 \mathrm{H}, \mathrm{s}, \mathrm{Me}_{\mathrm{b}}-4\right), 0.82(3 \mathrm{H}, \mathrm{s}, \mathrm{Me}-10), 0.86\left(3 \mathrm{H}, \mathrm{s}, \mathrm{Me}_{\mathrm{a}}-4\right), 0.95$ $(3 \mathrm{H}, \mathrm{d}, \mathrm{J}=6.6 \mathrm{~Hz}, \mathrm{Me}-13), 1.44$ (3H, s, Me-8), 1.93 (3H, s, OAc), 0.98-2.00 (16H, m, $\mathrm{H}-1,2,3,5,6,7 \mathrm{a}, 9,11,12,13), 2.12(1 \mathrm{H}, \mathrm{dd}, \mathrm{J}=14.7 \mathrm{~Hz}, 7.8 \mathrm{~Hz}, \mathrm{H}-14), 2.31(1 \mathrm{H}, \mathrm{dd}, \mathrm{J}=14.7 \mathrm{~Hz}, 6.5 \mathrm{~Hz}$, H'-14), 2.62 (1H, dt, J=12.5 Hz, 3.3 Hz, Hb-7), 3.66 (3H, s, OMe).

${ }^{13} \mathrm{C}$ NMR (75 MHz, $\left.\mathrm{CDCl}_{3}, \mathrm{~d}, \mathrm{ppm}\right): 39.48$ (C-1), 18.27 (C-2), 41.85 (C-3), 33.03 (C-4), 55.55 (C-5), 19.93 (C-6), 38.66 (C-7), 87.88 (C-8), 58.90 (C-9), 39.31 (C-10), 22.99 (C-11), 39.84 (C-12), 31.02 (C-13), 41.56 (C-14), 173.66 (C-15), 19.82 (C-16), 20.36 (C-17), 33.26 (C-18), 21.36 (C-19), 15.64 (C-20), 51.26 (OMe), 170.14 (OAc), 22.82 (OAc).

Acknowledgements: We wish to thank the Junta de Andalucía for financial support and the Ministerio de Educación, Cultura y Deporte for a Fellowship to J. M. Castro. 


\section{References and Notes}

1. Urones, J. G.; Basabe, P.; Marcos, I. S.; González, J. L.; Jiménez, V.; Sexmero, M. J.; Lithgow, A. M. Ambergris Compounds from Labdanolic Acid. Tetrahedron 1992, 48, 9991-9998.

Sample availability: Available from the authors and from MDPI

(C) 2003 MDPI. All rights reserved. 